REVIEW

\title{
Atrial pacing for the prevention of atrial fibrillation after coronary artery bypass graft surgery: a review of the literature
}

\author{
R A Archbold, R J Schilling
}

Heart 2004;90:129-133. doi: 10.1136/hrt.2003.015412

Atrial fibrillation (AF) occurs in $20-40 \%$ of patients after coronary artery bypass graft surgery (CABG) and contributes to increased morbidity and expenditure after CABG. The limited efficacy of pharmacological treatment to prevent post-CABG AF has stimulated research into alternative prophylactic strategies for the arrhythmia. This article critically reviews the trial evidence in the literature regarding the efficacy of epicardial atrial pacing to prevent post-CABG AF. Thirteen randomised controlled trials of either right, left, or biatrial pacing to prevent post-CABG AF were identified. Overall, prophylactic biatrial epicardial pacing appears to be effective prophylaxis against post-CABG AF and to reduce postoperative hospital stay. The efficacy of single site right or left atrial pacing is less clear. Further data are required to determine both the efficacy of single site atrial pacing and the cost effectiveness of pacing strategies to prevent $A F$ after CABG.

See end of article for authors' affiliations

Correspondence to:

DrR A Archbold

Department of Cardiology, London Chest Hospital, Bonner Road, Bethnall

Green, London E2 9JX, UK; Andrew.Archbold@ bartsandthelondon.nhs.uk

Accepted

10 September 2003
A pproximately 27000 people in the UK and 500000 people in the USA undergo coronary artery bypass graft surgery (CABG) each year, $20-40 \%$ of whom develop atrial fibrillation (AF) postoperatively. ${ }^{2}$ The arrhythmia contributes to increased morbidity and expenditure after $\mathrm{CABG}{ }^{3}$ Specifically, AF may cause haemodynamic compromise, postoperative stroke, and prolongation of hospital stay independent of other factors such as age. ${ }^{45}$

Current conventional clinical practice is dominated by therapeutic responses to the onset of post-CABG AF. Effective prophylaxis may, by contrast, be expected to produce corresponding reductions in morbidity and hospital stay, with resultant cost savings. Recognition of the potential benefits of preventing AF after CABG is reflected by the large number of prophylactic strategies reported in the literature. Only $\beta$ blockers, however, are widely used as prophylaxis. ${ }^{6}$ The need for a presurgery treatment period of 7-10 days limits the value of oral amiodarone. ${ }^{7}$ Furthermore, verapamil, diltiazem, and digoxin are not effective agents in preventing post-CABG AF. ${ }^{69}$ There are few data regarding the efficacy and safety of class I antiarrhythmic agents in this setting. ${ }^{10} 11$
The pathophysiology of post-CABG AF is incompletely understood. Patients predisposed to the arrhythmia have an electrophysiological substrate that can be identified preoperatively. ${ }^{2}$ The nature of this substrate may be slow atrial conduction or dispersion of atrial refractoriness. ${ }^{12}$ A number of perioperative factors, such as atrial extrasystoles, change in autonomic tone, electrolyte shifts, inadequate myocardial protection, $\beta$ blocker withdrawal, or the response to cardiopulmonary bypass, can trigger AF in these at-risk patients. ${ }^{13}$

The limited efficacy of pharmacological treatment to prevent post-CABG AF has stimulated research into alternative prophylactic strategies for the arrhythmia. Atrial pacing potentially may reduce bradycardia related AF and may favourably influence intra-atrial conduction, atrial refractoriness, and the frequency of atrial extrasystoles. We critically reviewed the trial evidence in the literature regarding the efficacy of epicardial atrial pacing to prevent post-CABG AF.

\section{METHODS}

The literature was searched on Medline for English language articles published before March 2003. The search terms used were "AF after $\mathrm{CABG}$ ", "atrial pacing for $\mathrm{AF}^{\prime}$, and "atrial pacing to prevent $\mathrm{AF}^{\prime \prime}$. Abstract books from the major US and European annual scientific meetings from 1996 onwards were searched for relevant abstracts. Randomised, prospective studies with a control group were included. The primary end point was the incidence of postoperative AF. The secondary end point was duration of postoperative hospital stay.

\section{The trial evidence: study method}

Thirteen prospective, randomised, controlled trials (two published in abstract only) were identified for critical review (table 1). Study populations ranged from 21 to 161 patients. The majority of trials studied patients undergoing isolated CABG. In four trials up to $15 \%$ of patients underwent valve surgery, either alone or in combination with CABG. ${ }^{14-17}$ Trials compared control with either right atrial pacing (RAP), ${ }^{18-20}$ biatrial pacing (BAP), ${ }^{17} 2122$ or both RAP and BAP. ${ }^{162324}$ Two trials compared left

Abbreviations: $A F$, atrial fibrillation; $B A P$, biatrial pacing; $C A B G$, coronary artery bypass graft surgery; $\mathrm{Cl}$, confidence interval; LAP, left atrial pacing; NAP, no atrial pacing; OR, odds ratio; RAP, right atrial pacing 
Table 1 Incidence of AF after CABG in trials of prophylactic pacing

\begin{tabular}{|c|c|c|c|c|c|c|}
\hline \multirow[b]{2}{*}{ Study } & \multirow[b]{2}{*}{$\mathbf{n}$} & \multicolumn{4}{|c|}{ Incidence of postoperative AF } & \multirow[b]{2}{*}{ p Value } \\
\hline & & Control & RAP & LAP & BAP & \\
\hline Kurz $1999^{14}$ & $21^{*}$ & $22.2 \%$ & & & $50 \%$ & \\
\hline Greenberg $2000^{15}$ & $154 \dagger$ & $37.5 \%$ & $8 \%$ & $20 \%$ & $26 \%$ & $0.002 \ddagger$ \\
\hline Daoud $2000^{16}$ & $118 \S$ & $28 \%$ & $32 \%$ & & $10 \%$ & 0.02 \\
\hline Gerstenfeld $2001^{17}$ & 118 & $35 \%$ & & & $21 \%$ & 0.08 \\
\hline Schweikert $1998^{18}$ & 86 & $34 \%$ & $34 \%$ & & & 1.0 \\
\hline Blommaert $2000^{19}$ & 96 & $27 \%$ & $10 \%$ & & & 0.036 \\
\hline Chung $2000^{20}$ & 100 & $28.6 \%$ & $27.5 \%$ & & & 0.90 \\
\hline Orr $1998^{21}$ & 120 & $29.5 \%$ & & & $18.2 \%$ & NS \\
\hline Levy $2000^{22}$ & 130 & $38.5 \%$ & & & $13.8 \%$ & 0.001 \\
\hline Gerstenfeld $1999^{23}$ & 61 & $33 \%$ & $29 \%$ & & $37 \%$ & $>0.7$ \\
\hline Neumann $2001^{24}$ & 90 & $32 \%$ & $31 \%$ & & $16 \%$ & 0.31 \\
\hline Fan $2000^{25}$ & 132 & $41.9 \%$ & $33.3 \%$ & $36.4 \%$ & $12.5 \%$ & $<0.05^{* *}$ \\
\hline Goette $2002^{26}$ & 161 & $42 \%$ & $48 \%$ & $37 \%+\dagger$ & & NS \\
\hline
\end{tabular}

atrial pacing (LAP) with control, RAP, and BAP. ${ }^{15} 25$ One study compared control with RAP and pacing at Bachmann's bundle. ${ }^{26}$ One study did not state the site of pacing, which was assumed to be RAP. ${ }^{18}$ Pacing protocols were AAT mode, ${ }^{16212224}$ AAI mode at 96 pulses $/ \mathrm{min}^{26}$ AAI mode at 100 pulses/min, ${ }^{17}$ AAI mode set at $10-20$ beats above the intrinsic atrial rate, ${ }^{14} 152025$ DDD mode at a lower rate of 100 pulses/min, ${ }^{23}$ and atrial overdrive algorithms that paced the atrium consistently above the intrinsic atrial rate. ${ }^{18} 19$ Duration of pacing ranged from 24 hours to five days postoperatively and was not stated in one study. ${ }^{18}$

In all trials the arrhythmia end point was AF except in two, which specified the combination of AF or atrial flutter as the end point. ${ }^{20}{ }^{23}$ The duration of the arrhythmia to qualify as an end point varied from $>1$ minute to $>1$ hour. The requirement for treatment of the arrhythmia was included in some end point definitions. Intensity of monitoring for arrhythmia ranged from continuous telemetry as dictated by standard clinical care to pacemaker Holter monitoring. The follow up period ranged from a single 24 hour period on the second postoperative day to total in-hospital stay.

\section{THE TRIAL RESULTS}

The first four studies to be published (two in abstract only) all failed to find a significant difference in AF incidence between paced patients (whether BAP or RAP) and controls (table 1). ${ }^{14} 182123$ In one of these studies, the incidence of AF lasting $>30$ minutes was $18.2 \%$ with BAP compared with $29.5 \%$ in controls (not significant) (fig 1). ${ }^{21}$ There was, however, a significant reduction in the median duration of episodes of AF (12 v 48 hours; $\mathrm{p}=0.008$ ) in the paced group. Another of these trials that also compared BAP with control was aborted after the recruitment of only 21 patients of a planned 200 due to an excess incidence of AF (50\% v $22 \%$ ) in the BAP study group (fig 1). ${ }^{14}$ This excess was attributed to the development of sensing failure and asynchronous atrial stimulation.

In two further trials, 154 cardiac surgical patients (136 isolated $C A B G$ ) and 132 CABG patients received either no atrial pacing (NAP), RAP, LAP, or BAP for 72 hours $^{15}$ or five days, ${ }^{25}$ respectively, at $10-20$ pulses/min above the native heart rate. In the first study, AF occurred significantly less frequently in the RAP group than in controls ( $8 \% v 37.5 \%$; $\mathrm{p}=0.002)$, but the incidence of AF was not significantly reduced compared with control in the LAP $(20 \%)$ or BAP (26\%) groups. ${ }^{15}$ Furthermore, postoperative hospital stay was shorter ( $5.6 \vee 7.8$ days; $\mathrm{p}=0.001)$ in the RAP group than in controls (table 2). By contrast, in the second study there was a significant reduction in the incidence of postoperative AF in the BAP group (12.5\%) when compared with the other three groups (LAP 36.4\%, RAP 33.3\%, control 41.9\%; p < 0.05). ${ }^{25}$ Mean hospital stay was also shorter (7.0 $v 9.6$ days;

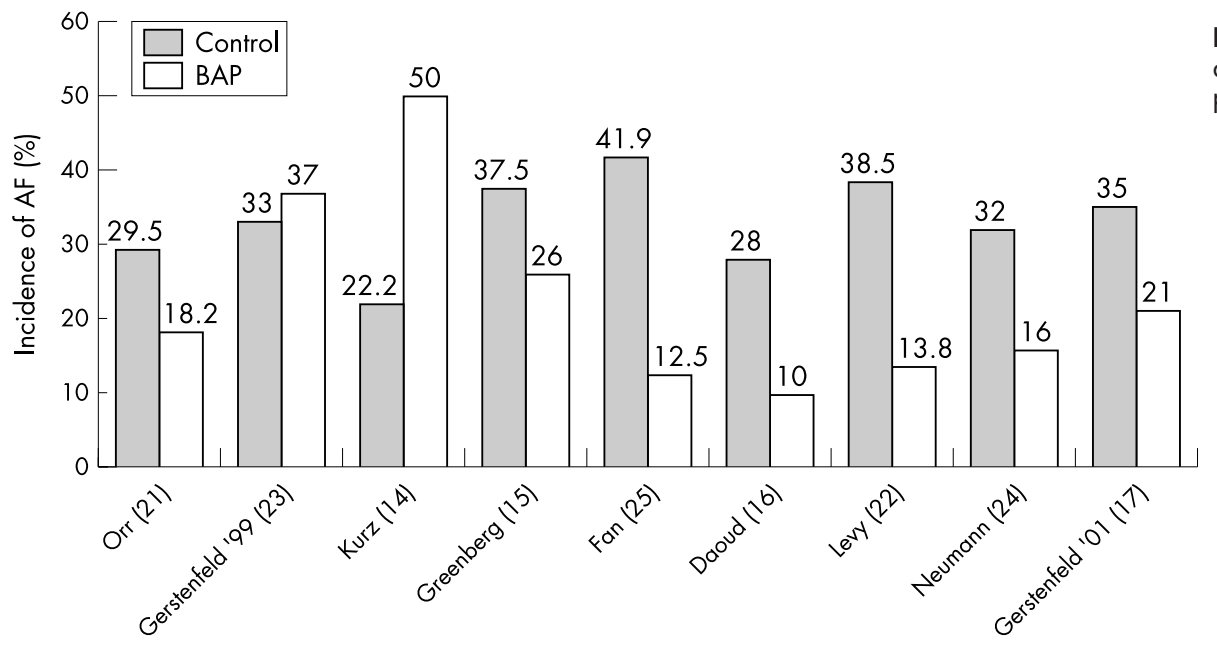

Figure 1 Incidence of postoperative atrial fibrillation $(A F)$ in trials of biatrial pacing (BAP).

Study (reference) 
Table 2 Duration of hospital stay after CABG in trials of prophylactic atrial pacing

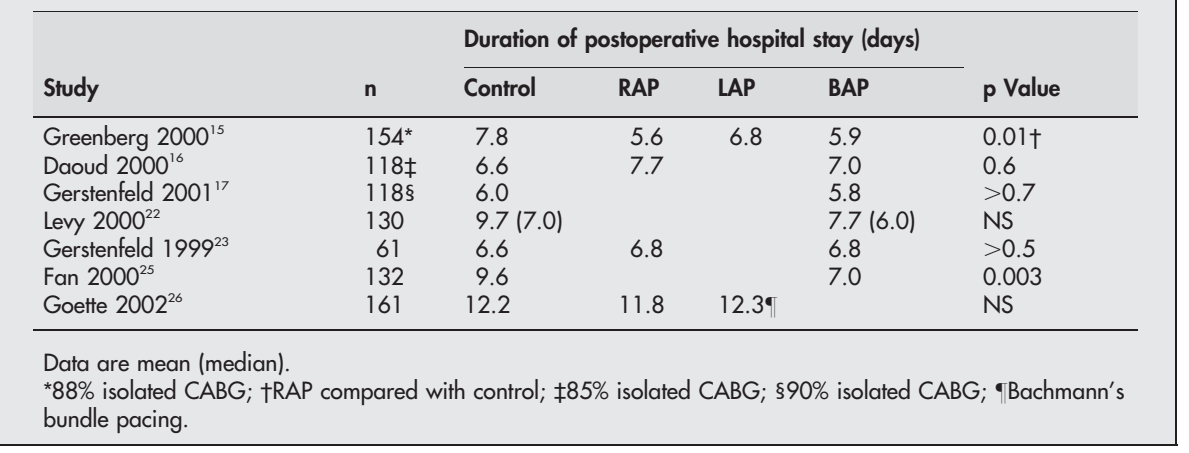

$p=0.003)$ in BAP patients than in controls. The incidence of AF was again significantly lower in the BAP group than in the RAP group (both AAT mode) and control (10\% v 28\% v $32 \%$, respectively; $p=0.02$ ) among 118 cardiac surgical patients. ${ }^{16}$ This did not translate into a reduction in hospital stay, however. Levy et $a l^{22}$ also showed that BAP reduced AF lasting $>1$ hour detected by pacemaker Holter monitoring from $38.5 \%$ to $13.8 \%(\mathrm{p}=0.001)$ and reduced AF detected clinically from $33.8 \%$ to $10.8 \%(\mathrm{p}=0.002)$. The mean and median hospital stays in the BAP group were 7.7 days and 6 days, respectively, compared with 9.7 days and 7 days in the control group (not significant).

Neumann $e a^{24}$ found that the incidence of AF detected by Holter monitoring during the first three days after CABG was lowest in patients randomised to AAT BAP (16\%), but this was not significantly different $(\mathrm{p}=0.31)$ from patients who received RAP $(31 \%)$ or NAP $(32 \%) .{ }^{24}$ Daoud et al ${ }^{16}$ randomly assigned 118 patients undergoing either CABG $(n=106)$, CABG plus aortic valve replacement $(n=5)$, or aortic valve replacement alone $(\mathrm{n}=7)$ to NAP or BAP in AAI mode at a rate of 100 pulses/min. The mean pacing period was 78 hours. AF lasting $>10$ minutes on Holter monitoring occurred in $21 \%$ of patients in the BAP group compared with $35 \%$ of patients in the NAP group $(\mathrm{p}=0.08)$. When patients undergoing $\mathrm{CABG}$ with or without aortic valve replacement ( $\mathrm{n}=111$ ) were considered, the incidence of AF was significantly lower in the BAP group ( $19 \%$ v 35\%; p $<0.05$; odds ratio (OR) $0.38,95 \%$ confidence interval (CI) 0.15 to $0.93)$. There was also a significant reduction in the amount of time spent on the intensive care unit among patients receiving BAP ( $37 v 50$ hours; $\mathrm{p}<0.05$ ) but the total hospital stay was similar in the two groups ( $139 v 143$ hours; $\mathrm{p}>0.7$ ).

Among 96 CABG patients randomly assigned to either RAP for 24 hours on the second postoperative day or to control, AF (during the second postoperative day) was reduced in the paced group compared with the control group (10\% v 27\%; $\mathrm{p}=0.036) .{ }^{19}$ Whether the overall incidence of in-hospital AF was reduced was not reported. By contrast, however, when Chung et al ${ }^{20}$ randomly assigned 100 patients undergoing CABG to RAP at 10 pulses/min above the native heart rate for four days postoperatively or to control, there was no difference in the AF frequency between groups $(25.5 \% \mathrm{v}$ $28.6 \%, p=0.90$ at four days; and $27.5 \%$ v $28.6 \%, p=0.90$ at seven days).

Most recently, Goette et $a^{26}$ randomly allocated 161 patients undergoing CABG to control, RAP, or pacing at Bachmann's bundle. The incidence of AF was not reduced by pacing at either site $(42 \% \vee 48 \% \vee 37 \%$, respectively; not significant). The length of hospital stay was also not influenced by either pacing strategy ( $12.2 v 11.8 v 12.3$ days, respectively; not significant), albeit in a health care system that tends to discharge patients later after CABG than in UK practice.

\section{DISCUSSION}

Pharmacological treatment has limited effectiveness for the prevention of post-CABG AF. Atrial overdrive pacing is effective at preventing AF in selected non-surgical patients with or without the need of a pacemaker. ${ }^{27}$ These factors together have stimulated considerable recent interest in atrial pacing as a preventive measure against post-CABG AF. We have reviewed the literature regarding its efficacy in preventing this arrhythmia.

We identified 13 randomised, controlled trials of prophylactic pacing for post-CABG AF. Several criticisms of these trials can be made. They had different exclusion criteria and the use of concomitant drugs, particularly $\beta$ blockers, varied, as did the intensity of monitoring for AF. Invariably they had low statistical power to detect a treatment effect. Despite the differences in study methods, two meta-analyses have pooled data from 10 (1473 patients) and 12 (1407 patients) randomised trials, respectively. ${ }^{28} 29$ In the former metaanalysis, the respective odds ratios (95\% confidence intervals) for AF for paced patients compared with a control group were as follows: RAP, OR $0.68,95 \%$ CI 0.39 to 1.19 ; LAP, OR $0.57,95 \%$ CI 0.28 to 1.16 ; and BAP, OR $0.46,95 \%$ CI 0.30 to 0.71 . These odds ratios were essentially the same as those reported in the second meta-analysis (published in abstract only). The results of meta-analyses should be interpreted with caution but these data suggest that BAP, at least, is an effective preventive measure against post-CABG AF. Furthermore, data from trials that randomly assigned 744 patients to BAP or control showed a significantly reduced mean length of hospital stay by 1.54 days (95\% CI -2.85 to -0.24 ), favouring pacing. ${ }^{28}$ Whether this reflects the reporting of hospital stay only in trials tending to show a benefit in paced patients is unclear.

The studies used a number of different pacing modalities (AAT, AAI, DDD, atrial overdrive algorithms) and different stimulation rates. It is probable that the effectiveness of atrial pacing in preventing AF after CABG is influenced by the pacing protocol in addition to the chamber(s) paced. BAP appears to be more effective than RAP or LAP, but the available data do not allow a definite conclusion to be drawn regarding the relative efficacies of pacing modality. Additional questions that remain unanswered relate to the feasibility, mechanism of benefit, and cost effectiveness of atrial pacing to prevent post-CABG AF.

\section{Feasibility}

The duration of effective pacing that was actually achieved varied considerably between studies. For example, BAP was successfully maintained in all cases to 96 hours with no complications in one study, ${ }^{21}$ whereas in two other studies BAP was discontinued before the 72 hour protocol in 33\% patients ${ }^{15}$ and in nine of 12 patients. ${ }^{14}$ Furthermore, successful pacing was achieved for a significantly shorter duration with the apparently more effective strategy of BAP than with 
RAP,${ }^{16}$ presumably because of its dependency on the integrity of two pairs of wires rather than one. Survival of the right atrial leads was significantly greater than for the left atrial leads $(\mathrm{p}<0.001)$. In their BAP patients, Gerstenfeld et $a l^{17}$ found that the left atrial wires had a significantly higher mean threshold than the right atrial wires, though no patients lost capture due to a large increase in threshold. Levy et al ${ }^{22}$ reported lead failure in $20 \%$ of their BAP patients, evenly split between left and right atrial wires. In the study of Goette et al, ${ }^{26}$ atrial pacing was terminated early in $6(10 \%)$ of 60 patients in the RAP group and in $3(5.9 \%)$ of 51 patients in the Bachmann's bundle pacing group. Pacing thresholds were significantly higher in the RAP group than in the Bachmann's bundle group $(\mathrm{p}<0.05)$, but there was no difference in atrial sensing. In the majority of cases, pacing was discontinued prematurely because of raised pacing thresholds, sensing failure, or diaphragmatic pacing. Such problems with the maintenance of the pacing regimens might have contributed to an underestimate of the efficacy of pacing, but they also raise issues about the feasibility of routine atrial pacing after $\mathrm{CABG}$.

The major complication of atrial pacing other than lead failure is cardiac tamponade after removal of the epicardial wires. This is rare, particularly in non-anticoagulated patients, and no such complications were reported in any of the trials. Nevertheless, at our institution epicardial pacing wires are removed before the day of discharge and with a surgeon available to perform open pericardial drainage when necessary.

\section{Potential mechanisms of benefit}

The mechanisms behind any reduction in the incidence of postoperative AF due to atrial pacing are speculative. Experiments in animals and humans suggest that re-entry is the underlying electrophysiological mechanism in the majority of cases of AF. ${ }^{12}{ }^{30}$ Persistence of AF requires that the depolarising wave fronts must continuously encounter excitable tissue, a circumstance favoured by slow atrial conduction. ${ }^{31} \mathrm{AF}$ is characterised by re-entry circuits propagating around areas of either fixed or functional conduction block. Functional block is dependent on heterogeneities of cardiac tissue ${ }^{32}$ that may be caused by factors such as oedema due to manipulation of the heart at surgery, atrial ischaemia, $^{33}$ changes in autonomic tone, ${ }^{34}{ }^{35}$ and inflammatory changes due to pericarditis or cardiopulmonary bypass. ${ }^{13}$ Intra-atrial conduction and refractoriness may be modified in a favourable way by atrial pacing. In patients with intraatrial conduction delay, for example, $\mathrm{P}$ wave duration was significantly decreased ( $106 v 187 \mathrm{~ms} ; \mathrm{p}<0.0001$ ) by BAP. ${ }^{36}$ In the study of Fan et $a{ }^{25}$ the mean $\mathrm{P}$ wave duration and mean $\mathrm{P}$ wave dispersion were significantly reduced with atrial pacing irrespective of pacing site. The percentage reduction in mean $\mathrm{P}$ wave duration was similar in the three pacing groups but BAP resulted in a significantly greater reduction in mean $\mathrm{P}$ wave dispersion. By contrast, however, RAP prolonged $\mathrm{P}$ wave duration in the studies of Blommaert et $a l^{19}$ and Goette et $a l^{26}$ and prolongation of $\mathrm{P}$ wave duration during RAP was an independent predictor for postoperative $\mathrm{AF}$ in the latter study. ${ }^{26}$

Atrial pacing may be expected to reduce bradycardia related AF. The presence of atrial extrasystoles preoperatively independently predicts the development of post-CABG AF. ${ }^{37}$ Furthermore, the frequency of atrial extrasystoles increases before the onset of $\mathrm{AF}$ after $\mathrm{CABG}^{34}{ }^{35}$ and they are a common initiating factor for the arrhythmia. ${ }^{19}{ }^{23}$ Overdrive atrial pacing algorithms have been shown to decrease the frequency of premature atrial complexes. ${ }^{39}{ }^{40}$ However, Chung et $a l^{20}$ found that not only was the number of atrial extrasystoles increased in the control patients who developed
AF (2715 v 232 in 24 hours; $\mathrm{p}=0.023)$, but they were also increased in paced compared with non-paced patients $(2106 \mathrm{v}$ 866 in 24 hours; $p=0.0001)$. Chung et $a^{20}$ concluded that atrial pacing in the postoperative period may actually be proarrhythmic. In this study, only the right atrium was paced, a strategy that may result in less favourable changes in intra-atrial conduction than with BAP. These contrasting findings highlight the limitations of our current understanding of the pathophysiology of post-CABG AF.

\section{Conclusion}

Prophylactic biatrial epicardial pacing appears to be effective prophylaxis against post-CABG AF but the effectiveness of single site RAP or LAP is less clear. It is not known whether (bi)atrial pacing is a cost effective strategy to prevent postCABG AF. If the significant reduction in mean length of hospital stay of 1.54 days seen in patients randomly assigned to BAP in the trials can be reproduced in "real world" clinical practice, this approach to AF may reduce overall cost per patient. Further data are required to determine both the effectiveness of single site atrial pacing and the cost effectiveness of pacing strategies to prevent AF after CABG.

\section{Authors' affiliations}

R A Archbold, Department of Cardiology, London Chest Hospital, London, UK

R J Schilling, Department of Cardiology, St Bartholomew's Hospital, London, UK

\section{REFERENCES}

1 Cresswell LL, Schuessler RB, Rosenbloom M, et al. Hazards of post-operative atrial arrhythmias. Ann Thorac Surg 1993;56:539-49.

2 Zaman AG, Archbold RA, Helft G, et al. Atrial fibrillation after coronary artery bypass surgery: a model for pre-operative risk stratification. Circulation 2000;101:1403-8.

3 Archbold RA, Curzen N. Atrial fibrillation after coronary artery bypass graft surgery: more than an irritation! Clin Intensive Care 1999;10:109-16.

4 Borzak S, Tisdale JE, Amin NB, et al. Atrial fibrillation after bypass surgery: does the arrhythmia or the characteristics of the patients prolong hospital stay? Chest 1998;113:1489-91.

5 Tamis JE, Steinberg JS. Atrial fibrillation independently prolongs hospital stay after coronary artery bypass surgery. Clin Cardiol 2000;23:155-9.

6 Andrews TC, Reimold SC, Berlin JA, et al. Prevention of supraventricular arrhythmias after coronary artery bypass surgery: a meta-analysis of randomized control trials. Circulation 1991;84(suppl III):236-44.

7 Zaman AG, Archbold A, Alamgir F. Amiodarone prophylaxis for atrial fibrillation after cardiac surgery. N Engl J Med 1998:338:1383-4.

8 Kowey PR, Taylor JE, Rials SJ, et al. Meta-analysis of the effectiveness of prophylactic drug therapy in preventing supraventricular arrhythmia early after coronary artery bypass grafting. Am J Cardiol 1992;69:963-5

9 Babib-Ebell J, Keith PR, Elert O. Efficacy and safety of low-dose propranolol versus diltiazem in the prophylaxis of supraventricular tachyarrhythmia after coronary artery bypass grafting. Eur J Cardiothorac Surg 1996;10:412-6.

10 Laub GW, Janeira L, Muralidharan S, et al. Prophylactic procainamide for prevention of atrial fibrillation after coronary artery bypass grafting: a prospective, double-blind, randomized, placebo-controlled pilot study. Crit Care Med 1993;21:1474-8.

11 Gold MR, O'Gara PT, Buckley MJ, et al. Efficacy and safety of procainamide in preventing arrhythmias after coronary artery bypass surgery. Am J Cardiol 1996;78:975-9.

12 Cox JL, Schuessler RB, Boineau JP. The surgical treatment of atrial fibrillation I: summary of the current concepts of the mechanisms of atrial flutter and fibrillation. J Thorac Cardiovasc Surg 1991;101:402-5.

13 Archbold RA, Curzen NP. Off-pump coronary artery bypass graft surgery: the incidence of postoperative atrial fibrillation. Heart 2003;89:1134-7.

14 Kurz DJ, Naegeli B, Kunz M, et al. Epicardial, biatrial synchronous pacing for prevention of atrial fibrillation after cardiac surgery. Pacing Clin Electrophysiol 1999;22:721-6.

15 Greenberg MD, Katz NM, luliano S, et al. Atrial pacing for the prevention of atrial fibrillation after cardiovascular surgery. J Am Coll Cardiol 2000;35:1416-22.

16 Daoud EG, Dabir R, Archambeau M, et al. Randomized, double-blind trial of simultaneous right and left epicardial pacing for prevention of post-open heart surgery atrial fibrillation. Circulation 2000;102:761-5.

17 Gerstenfeld EP, Khoo M, Martin RC, et al. Effectiveness of bi-atrial pacing for reducing atrial fibrillation after coronary artery bypass graft surgery. J Interv Card Electrophysiol 2001;5:275-83.

18 Schweikert RA, Grady TA, Gupta N, et al. Atrial pacing in the prevention of atrial fibrillation after cardiac surgery: results of the second post operative pacing study (POPS-2) [abstract]. J Am Coll Cardiol 1998;31(suppl A): $117 \mathrm{~A}$. 
19 Blommaert D, Gonzalez M, Mucumbitsi J, et al. Effective prevention of atrial fibrillation by continuous atrial overdrive pacing after coronary artery bypass surgery. J Am Coll Cardiol 2000;35:1411-5.

20 Chung MK, Augostini RS, Asher CR, et al. Ineffectiveness and potential proarrhythmia of atrial pacing for atrial fibrillation prevention after coronary artery bypass grafting. Ann Thorac Surg 2000;69:1057-63

21 Orr WP, Tsui S, Stafford PJ, et al. Synchronised bi-atrial pacing for the prevention of atrial fibrillation after coronary artery bypass surgery: a feasibility study [abstract]. Heart 1998:79(suppl 1):6.

22 Levy T, Fotopoulos G, Walker S, et al. Randomized controlled study investigating the effect of biatrial pacing in prevention of atrial fibrillation after coronary artery bypass grafting. Circulation 2000;102:1382-7.

23 Gerstenfeld EP, Hill MR, French SN, et al. Evaluation of right atrial and biatrial temporary pacing for the prevention of atrial fibrillation after coronary artery bypass surgery. J Am Coll Cardiol 1999;33:1981-8.

24 Neumann T, Sperzel J, Ehrlich W, et al. Biatrial pacing after CABG surgery: prevention of atrial fibrillation? Europace Suppl 2001;2:B202.

25 Fan K, Lee KL, Chiu CSW, et al. Effects of biatrial pacing in prevention of postoperative atrial fibrillation after coronary artery bypass surgery. Circulation 2000; 102:755-60.

26 Goette A, Mittag J, Friedl A, et al. Pacing of Bachmann's bundle after coronary artery bypass grafting. Pacing Clin Electrophysiol 2002;25:1072-8.

27 Lau C-P. Pacing for atrial fibrillation. Heart 2003;89:106-12.

28 Crystal E, Connolly SJ, Sleik K, et al. Interventions on prevention of postoperative atrial fibrillation in patients undergoing heart surgery: a metaanalysis. Circulation 2002;106:75-80.

29 Pinski SL, Sgarbossa EB. Influence of overdrive atrial pacing on the occurrence of atrial fibrillation after cardiac surgery: a meta-analysis [abstract]. J Am Coll Cardiol 2001;37(suppl A):128A.

30 Konings KT, Kirchof CJ, Smeets JR, et al. High-density mapping of electrically induced atrial fibrillation in humans. Circulation 1994;89:1665-80.
31 Levy S, Breithardt G, Campbell RWF, et al, on behalf of the Working Group on Arrhythmias of the European Society of Cardiology. Atrial fibrillation: current knowledge and recommendations for management. Eur Heart $J$ 1998; 19:1294-320.

32 Scheinman MM. Mechanisms of atrial fibrillation: is a cure at hand? J Am Coll Cardiol 2000;35: 1687-92.

33 Tchervenkov $\mathrm{Cl}$, Wynands JE, Symes JF, et al. Persistent atrial activity during cardioplegic arrest: a possible factor in the etiology of postoperative supraventricular tachyarrhythmias. Ann Thorac Surg 1983;36:437-43.

34 Dimmer C, Tavernier R, Gjorgov N, et al. Variations in autonomic tone preceding onset of atrial fibrillation after coronary artery bypass grafting. Am J Cardiol 1998;82:22-5.

35 Hogue CW Jr, Domitrovich PP, Stein PK, et al. RR interval dynamics before atrial fibrillation in patients after coronary artery bypass graft surgery. Circulation 1998;98:429-34.

36 D'Allonnes GR, Pavin D, Leclercq C, et al. Long-term effects of biatrial synchronous pacing to prevent drug-refractory atrial tachyarrhythmias: a nine-year experience. J Cardiovasc Electrophysiol 2000;11:1081-91.

37 Frost L, Molgaard H, Christiansen EH, et al. Low vagal tone and supraventricular ectopic activity predict atrial fibrillation and flutter after coronary artery bypass grafting. Eur Heart J 1995;16:825-31.

38 Jidéus L, Blomström P, Nilsson L, et al. Tachyarrhythmias and triggering factors for atrial fibrillation after coronary artery bypass operations. Ann Thorac Surg 2000;69:1064-9.

39 Ricci R, Santini M, Puglisi A, et al. Impact of consistent atrial pacing algorithm on premature atrial complex number and paroxysmal atrial fibrillation recurrences in brady-tachy syndrome: a randomised prospective cross over study. J Interv Card Electrophysiol 2001;5:33-44.

40 Lam CT, Lau CP, Leung SK, et al. Efficacy and tolerability of continuous overdrive atrial pacing in atrial fibrillation. Europace 2000;2:286-91.

\section{IMAGES IN CARDIOLOGY}

\section{Spontaneous termination of ventricular fibrillation}

A 65 year old woman with hypertension and permanent atrial fibrillation had suffered two brief syncopal episodes. She was on losartan, digoxin, and aspirin. The ECG showed atrial fibrillation and incomplete left bundle branch block; the QT interval was $0.36 \mathrm{~s}$ and the QTc 0.414 s. The echocardiogram revealed mild dilatation and dysfunction of the left ventricle (ejection fraction $=40 \%$ ). Laboratory tests were normal; serum potassium was $4.1 \mathrm{mEq}$, magnesium $2.2 \mathrm{mEq}$, and digoxin $0.95 \mathrm{ng} / \mathrm{ml}$.

The Holter recording (right) revealed an episode of ventricular fibrillation (VF) occurring during sleep. Surprisingly, VF ceased after 1.16 mins; an asystolic pause of $16.08 \mathrm{~s}$ then ensued, followed by resumption of a supraventricular rhythm.

Ventricular fibrillation seldom terminates spontaneously, since several re-entrant wavefronts, independent from each other, coexist, and the simultaneous extinction of all the circuits is unlikely. In the present case, fibrillation waves are initially tall and regular, and later become irregular and reduced in voltage (third strip). From the second half of the fourth strip, however, the tracing shows again regular and high voltage waves. Although the term "ventricular fibrillation" is appropriate to describe this rhythm disorder, fragmentation of the electrical activity, as deduced from the ECG, is not very pronounced, to the point that in the section preceding termination the pattern resembles a fast ventricular tachycardia rather than a true VF. This may help to explain the unexpected spontaneous interruption of the arrhythmia.

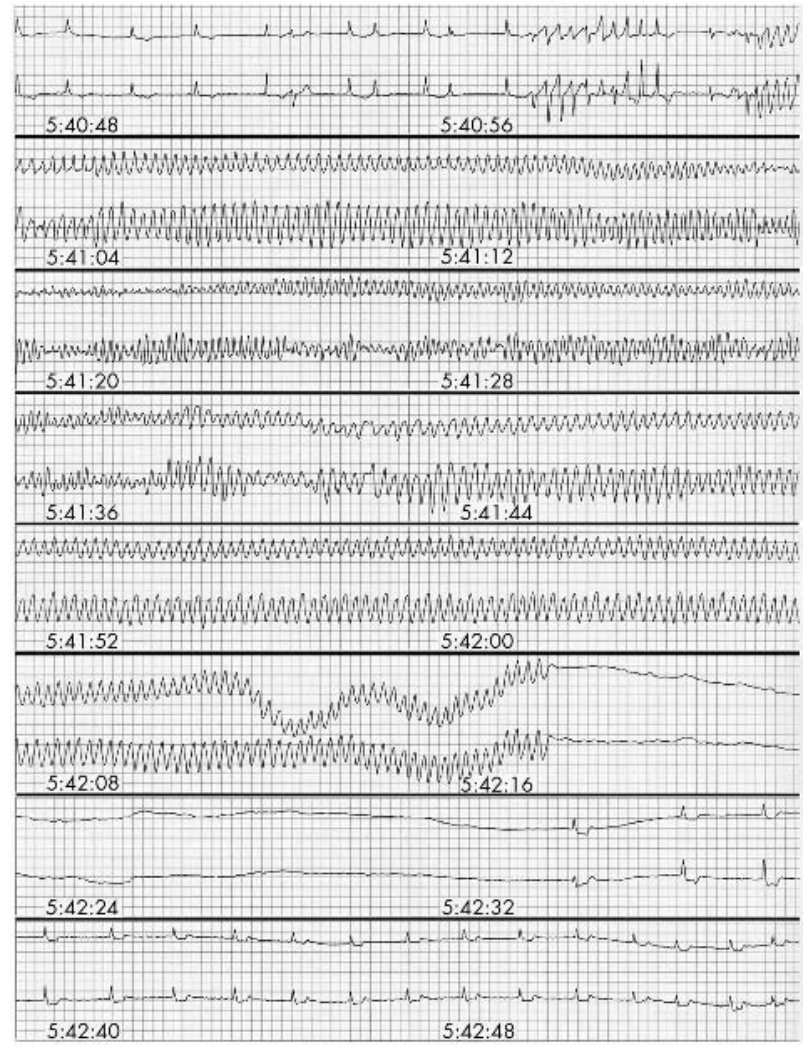

A continuous two channel Holter recording. The top strip shows a short run of torsades de pointes; following the post-tachycardia pause, a single narrow beat occurs, and then a premature ventricular complex initiates ventricular fibrillation. 